\title{
DENOMINACIONES GEOGRÁFICAS: MÁS QUE INSTRUMENTOS COMERCIALES
}

\author{
Alonso Rodríguez Chaves \\ Escuela de Ciencias Sociales \\ Universidad Estatal a Distancia, Costa Rica \\ arodriguez@uned.ac.cr
}

\section{RESUMEN}

El artículo hace referencia a la importancia que han adquirido las denominaciones geográficas en los últimos años, principalmente porque como instrumentos comerciales han incidido en el favorecimiento y promoción de productos con peculiaridades territoriales y culturales. En consecuencia, la tesis transversal presentada en el artículo, se basa explícitamente en algunas experiencias, cuya aplicación sobrepasa los efectos en el ámbito comercialy destacan como coadyuvadores del desarrollo rural integral. Así se invita al lector a conocer el impacto que han tenido como proyectos productivos culturales, en tanto, la promoción y valorización de recursos, no solo aproximan a comunidades desfavorecidas rurales a la sostenibilidad y perdurabilidad económica sino también a una mayor articulación socialy valoración de su cultura.

PALABRAS CLAVE: DENOMINACIONES GEOGRÁFICAS, TERROIR O TERRUÑO, PROMOCIÓN COLECTIVA, DESARROLLO RURAL, CULTURA EMPRESARIAL Y TRAZABILIDAD

\section{ABSTRACT}

This article makes reference to the importance that geographic denominations have acquired in the last years, mainly because the have influenced in the enhancement and promotion of products with cultural and territorial peculiarities as commercial instruments. As a consequence, the transversal thesis presented in the article, is explicitly based on some experiences, which application exceed the effects in the commercial field and stand out as coadyuvants in the integral rural develop- ment. Therefore, invite the reader to know the impact they have had as cultural productive projects. The promotion and the value of resources, not only encourage the rural disadvantaged communities to a economical sustainable and lasting state but also to a major social articulation and appreciation of their culture.

KEYWORDS: GEOGRAPHICAL DENOMINATIONS, TERROIRORNATIVE LAND, COLLECTIVE PROMOTION, RURAL DEVELOPMENT, ENTERPRISE CULTURE AND OUTLINING.

\section{INTRODUCCIÓN}

Uno de los rasgos más sobresalientes del mundo en la segunda mitad del siglo XX, fue compensar el desequilibrio territorial e infrautilización de recursos naturales y ambientales. Así para enmendar dichos problemas se concede alto aprecio y valor al conjunto de intereses y capacidades propias que las comunidades rurales poseen.

Bajo esa lógica, se tiende a favorecer el uso de denominaciones geográficas, como instrumentos comerciales que valorizan el prestigio de productos por peculiaridades territoriales y culturales.

En consecuencia, el artículo asume como objeto de estudio, el efecto y significado que adquieren dichos instrumentos en el desarrollo rural. Por lo que se invita al lector a conocer el impacto que han tenido en la promoción y valorización de recursos, entre otros beneficios reconocidos; que aproximan a comunidades desfavorecidas a la sostenibilidad y perdurabilidad ecológica, económica y social en general. 


\section{ANTECEDENTES}

En el transcurso de la historia, lo típico adquiere alta carga simbólica y ayuda a conformar el imaginario colectivo de diferentes sociedades, especialmente cuando se relacionan con el arraigo a la tierra y la cultura. Así las cosas, las denominaciones geográficas significan valiosos instrumentos para el desarrollo y la "promoción colectiva", al ser fruto de la relación estrecha establecida entre grupos humanos y la tierra de donde provienen. Como señala Bérard y Marchenay (1996): "Valorar los productos de nuestra tierra, es valorar también nuestros conocimientos técnicos y las riquezas portadoras de nuestras tradiciones y culturas" (p. 35).

De esta manera, el concepto acuñado de las denominaciones geográficas no solo se debe relacionar con el territorio "terroir" o "terruño", sino con la interacción de múltiples variables que intervienen, para valorizar el prestigio de productos. En particular se anotan las siguientes dimensiones (Bérard \& Marchenay, 1996).

- Geográfica, porque el territorio es donde se origina el producto. Destacando elementos geográficos de su lugar de origen.

- Cultural, porque en el territorio se vincula la producción a costumbres. A pesar de la ambigüedad, su condición es otorgada por el método de producción arraigado en la tradición y culturalmente aceptada en la comunidad que trate.

- Histórica, porque en el territorio se asienta en el tiempo, prácticas, métodos, técnicas y conocimientos sobre el producto. Lo cual implica plusvalía cualitativa ligada a la continuidad, la duración o permanencia.

En vista de ello, la Organización Internacional de la Viña y el Vino (OIV) considera esta triple dimensión, como elementos vitales, que permiten dar alta valorización y distinguir el producto de otros similares, sin que tenga que haber necesariamente connotación valorativa de excelencia. Como resultado, en Europa se han catalogado típicos, tradicionales o de la tierra, unos 6000 productos, que represen- tan el 11,6\% del volumen del mercado alimentario total en Italia; el 10,6\% en Francia; el 8,1\%; en Grecia; el 6,1\%; tanto en España como en Bélgica; el 5,2\% y en Portugal; el 3,1\% (Renamur, 2006).

Por ende, no es extraño el interés creciente de la Red Internacional de Indicaciones Geográficas conformada por productores de 30 países en promoverlas, pues según se aprovechan capacidades, recursos y aspiraciones comunes, que se traducen en elementos de promoción, valorización de recursos locales y mejora económica de zonas rurales (Colom, Escardíbul, Fransi \& Saez, 1999).

\section{Implicaciones directas}

Así los rasgos mencionados pasan a conformar el sentido de las Denominaciones Geográficas Protegidas y puntos de referencia para encontrar nuevos papeles a la ruralidad. En tanto, dichas experiencias, apuntan a ser sumamente beneficiosas para productores rurales. A través de esas acciones, las comunidades se aproximan a las siguientes situaciones (Martínez \& Jiménez, 1999).

\section{Protege el valor local}

Las distinciones geográficas están ligadas al territorio, por ende, el producto tiene que provenir de un sitio determinado. Resultado del vínculo, la protección evita la deslocalización de la producción y son probablemente, la única forma de propiedad intelectual que comunidades sean susceptibles de poseer. En consecuencia, se convierte en un derecho colectivo ideal, que no necesariamente requiere de innovación; solo ofrecer que el producto sea del lugar con métodos de producción particulares (Thual, s.f).

Esto, confirma el interés de la titularidad o "propiedad" de las denominaciones geográficas, lo cual constituye un aspecto importante para su propio desarrollo y promoción; así como de interés de los productores en obtener distinción, puesto que los productos de un lugar, inciden en el fortalecimiento de la posición de los productos autóctonos. 


\section{Diversifica la producción}

Entendida la diversificación como la necesidad de cambiar la oferta productiva tradicional, supuestamente por productos más rentables, esto ha influido en la seguridad alimentaria, cuando se presentan desabastecimientos de vitales productos. Bajo idea de enmendar la situación, en julio de 1992, la Comunidad Europea adoptó en su política de desarrollo rural, promover la protección de denominaciones geográficas en sectores agrícolas y relacionados (Downes \& Laird, 1997).

Por consiguiente, la nueva política de desarrollo rural reconoce importante, potencializar y sostener la oferta productiva tradicional, manteniéndose cada región en lo que históricamente sabe mejor hacer. Así se garantiza un equilibrio entre oferta y demanda, con productos de calidad y variedad.

En ese sentido, se refieren a las denominaciones geográficas como "la fuerza de la diferencia", que vistas como fortalezas y aprovechadas pueden llegar a ser vitales en áreas marginales, que no pueden competir en otros productos. Elena Sarraceno (2000), señala, que la heterogeneidad de áreas rurales debe ser reconocida como elemento positivo. Bajo esa lógica, las denominaciones geográficas tienen un rol decisivo para mejorar la utilización de tierras y la gestión de recursos de zonas; lo cual diversifica la producción de comunidades rurales y la seguridad alimentaria (Comisión Europea, 2006)

\section{Fomenta la cultura empresarial}

El Sistema Agroalimentario europeo se ha caracterizado en los últimos años, por estar sometido a impactos de intervención supranacional, que persiguen desarrollar una cultura empresarial en la que han comprendido la necesidad de pertenecer a un sistema socio-organizacional conformado por personas o grupos con las que establece relaciones formales e informales, para armonizar intereses y aspiraciones comunes (Colom et al., 1999).

Con la experiencia, se entiende que la unión crea relaciones, se transforma en conjunto social uni- tario y coherente para perseguir un fin, con sentido y eficacia, ya que la unidad organizacional establece relaciones de poder con otros agentes. Como ejemplo se presenta la región autónoma de Galicia, España, históricamente caracterizada por su ruralidad y la amplia gama de productos de prestigio que han rebasado fronteras, gracias en parte, a labor tesonera de campesinos emigrantes, quienes actuaron como propagadores del patrimonio gastronómico-cultural (Comunidad Autónoma de Galicia, 2005).

\section{Añade valor a las tierras}

Las Indicaciones Geográficas proporcionan valor añadido a las tierras que ostentan protección en sus productos. Un estudio realizado por "huile d'olive de Nyons" (Aceite de oliva) muestra como resultado de la protección, el valor de la tierra dedicada a los olivos destinados a esa producción, lo cual aumentó un 2\%, más rápido que el de la tierra destinada a producción desprotegida (Thual, s.f.).

En ese sentido, las estrategias por conservar y rescatar tierras hacen de las comunidades más dinámicas, a la vez, codiciadas y aptas para invertir. Es así como diversas formas de agricultura con distinciones geográficas, no solo deben ser relacionadas como simples herramientas para producir y mantener productos con ciertas características sino como coadyubadoras a dar plusvalía a las tierras. En el caso europeo, varias localidades miradas antes con menosprecio, hoy se tratan de localidades diferentes y con potencial para desarrollar importantes proyectos de producción con protección (Ferrari, 2008).

Por ende, se han convertido en herramientas favorecedoras para la proliferación de empresas gestoras de nuevos proyectos y redistribución de actividad económica. Aún a sabiendas del coste en la implantación, el balance beneficio-coste es favorable, contribuyendo con inversiones que buscan el equilibrio entre territorios, aumento del nivel de vida y desarrollo. En particular nos referimos a medidas que valorizan recursos humanos, inversiones en favor de la educación, la sanidad 
e higiene pública, el aprovisionamiento y mejora de infraestructuras de comunicación, viales y de telecomunicación, entre otros servicios, que carecían las comunidades (Ferrari, 2008).

\section{Genera actividades económicas paralelas}

Entre las implicaciones directas de las denominaciones geográficas, destaca la generación de actividades económicas paralelas. El turismo constituye parte de un filón de opciones para el desarrollo de negocios rurales con sus consecuentes efectos en la generación de empleo e ingresos (Riveros \& Blanco, 2003). En Italia, Francia y España se organizan rutas turísticas a comunidades rurales en que sus productos obstentan alguna denominación protegida, lo que contribuye positivamente a la dinámicas socio-económicas. La Ruta de Toro por ejemplo, pertenece a las rutas enoturísticas de Valladolid, España; en esta se incluye visitas a bodegas de la Denominación de Origen Toro, Ribera del Duero y Rueda (Oficina de Ecoturismo de Valladolid, s.f.).

Con la red de Paradores de Turismo y hospedajes en sitios históricos se ha puesto en marcha proyectos en que ofrecen itinerarios gastronómicos, para exaltar elementos de la cocina autóctona como el aceite de oliva extra virgen y el bacalao. La ocasión sirve para educar y dar información, sobre el origen de los alimentos y las regiones, pues se utilizan con fines didácticos, para contar la historia de sus predecesores, ritos y formas de alimentación popular de larga data (El Financiero, 2002).

Por consiguiente, el agroturismo constituye gran alternativa, en la que se benefician propietarios de esos emprendimientos y demás pobladores que erigen nuevas fuentes de ingresos y argumento adicional para arraigarse. Ejemplo de los alcances de la articulación agroindustria rural con turismo, destaca la Red de Descubrimiento del Mundo Rural, en la comarca de El Condado, en Andalucía, España, que ofrece posibilidad de conocer modos de producción y organización de empresas rurales (Riveros \& Blanco, 2003).

\section{Integra las comunidades rurales}

Otras implicaciones de las distinciones geográficas es la integración social; en cuanto las organizaciones de productores articulan jornadas de trabajo para la producción, transformación y comercialización, lo que conllevaba a realizar actividades integradas y ordenadas. Los frutos constan en la existencia de un tejido social territorial articulado, que sustenta modos de acción colectiva, capaces de generar mayor autonomía, equidad y autodeterminación en procesos de desarrollo en toda su diversidad (Llorente, 2001).

Ablan (2000) coincide, que la reputación de productos se debe en parte, a los elementos que participan en la cadena productiva y comercial; a la integración de la administración y gestión en la definición, implementación y control de las denominaciones. Él hace hincapié a la disciplina impuesta para los involucrados, con la que se obtiene beneficios y se mantiene el prestigio adquirido del producto. Bien resume que hay que valorar"desde la granja hasta la mesa"(Ablan, 2000, p. 63.)

El desarrollo efectivo de las denominaciones geográficas, favorecen la integración con el fin de garantizar y mantener el prestigio, tratando que se consiga en pie de igualdad entre operadores. En esa dirección, nuevas oportunidades y posibilidades surgen con novedosas orientaciones, al igual, la posibilidad de integrar actividades de transformación al nivel familiar artesanal o con carácter manufacturero o semi-industrial a través de modelos empresariales individuales o colectivos, incluyendo claro la función del comercio o distribución.

De no ser así, sería difícil la defensa y promoción de productos aislada, condición que supera el ámbito económico, para entroncar en aspectos socio-culturales.

Colom etal. (1999) consideran que las acciones en favor del desarrollo local que integran el conjunto de intereses en las comunidades rurales, favorece la capacidad de iniciativas a escala local.

\section{Aumento de la renta}

Mediante la diferenciación de los productos por su origen, los indicadores geográficos pueden aumentar el precio del bien o producto. Las ventajas se resumen en mayor renta para el productor al 
obtener mayores posibilidades de fijar el precio, lo que les ha permitido diferenciarse de competidores y blindarse de la volatilidad de los precios del producto (Gómez \& Caldentey, 2000).

Un estudio realizado en Francia señala que el precio entre productos con o sin denominación es de $30 \%$ para queso y de hasta $230 \%$ para vinos. Así en el 2002, el precio medio para litro de leche era de 0.30 mientras que la leche suministrada a los productores de queso con IG francesa Beaufort era de 0.57 el litro (+90\%) (Thual, s.f.).

De esta manera, el uso de las distinciones geográficas promete crecimiento de la demanda y precios rentables, más cuando las perspectivas del mercado mundial lo indican y cuando el $60 \%$ de la población de Estados miembros de la Unión Europea vive en zonas rurales, lo que representa el 90\% de su territorio (Jiménez, 2002).

\section{Define nuevas estrategias de mercado}

En países europeos de tradición vinícola se tiene documentado diferentes crisis de producción desde finales del siglo XIX. Con el afán de superarlas, los productores se han organizado para mejorar colocación y venta del producto, asociando el vino a su región de origen y su cultura (Avelino, Marie \& Ribeyre, 2007).

Hoy, ante un mundo en el que se genera creciente estandarización de los productos, con insuficiencias de calidad y trazabilidades, las denominaciones se aprecian como nichos diferenciales de originalidad y seguridad. En particular, para competir e insertarse en la creciente globalización y mundialización económica y cultural, reproducida por medios de comunicación, que convierten las grandes marcas y hábitos de consumo en referentes de pertenencia global (Llorente, 2001).

En este contexto, Martínez y Jiménez (1999) coinciden en señalar, que una de las cuestiones fundamentales que han incidido casualmente, es la creación de nuevas estrategias de mercado, más cuando el grado de diferenciación y saturación es elevado, por lo que se ha logrado reforzar y mejorar el reconocimiento de productos respecto a otros, ya que:

(...) la situación competitiva caracterizada por una estructura empresarial fuertemente diversificada, atomizada y con alto grado de rivalidad obliga a establecer la diferenciación sobre la base de una calidad determinada por el origen del producto, permitiendo así, el aprovechamiento de puntos fuertes derivados de: la tradición, la experiencia y el saber-hacer en la elaboración del producto que existe en una zona geográfica y en las empresas en ella instaladas. Obteniéndose importantes beneficios a través de la posición privilegiada que el producto ocupa en el mercado. (Martínez \& Jiménez, s.f, p.12).

Con relación a las características diferenciales de los productos, las posibles estrategias de comercialización y perspectivas de mercado, constituyen oferta distinta y sustantiva para el consumidor. Bajo este esquema, las ventajas competitivas que han brindado las características de un producto por su origen, por métodos de producción o elaboración, se han considerado elementos determinantes.

Gómezy Caldentey (1997) identifican el origen, como verdaderas estrategias de potenciación de productos autóctonos en los mercados, al considerarlas como nuevas vías de comercialización, ya que:

(...) descansa sobre algunos escasos elementos en que una determinada zona productora puede satisfacer al mercado y ser competitiva en actuales circunstancias: diferenciación, calidad y territorio. Pero no como factores que actúan independientemente, sino relacionados, es decir: se tiene calidad diferencial ligada al territorio (p. 12).

No obstante, la buena aceptación de estas herramientas, se percibe en la confluencia pragmática de consumidores "informados y conscientes" en proceso de selección. Al respecto, un estudio de la Unión Europea revela que un $76 \%$ de consumidores valora y adquiere productos fabricados de 
manera tradicional y un 43\% se fija en el etiquetado para identificar productos elaborados en forma tradicional. Además, cinco de cada diez europeos han oído hablar de las distinciones geográficas, cuatro de cada diez las compraban, y uno de cada dos europeos tiene confianza en los productos, siempre y cuando órganos correspondientes garanticen vínculo con el lugar determinado y modo tradicional de elaboración (Pérez, 1998).

Lo anterior, nos muestra a consumidores dispuestos a pagar un excedente por productos con estas características, lo que obliga a pequeños productores a mantener y mejorar su reputación, así de vender directamente al consumidor final, compitiendo así más eficiente con las grandes corporaciones.

La Comisión Europea, se ha empeñado que los productores aporten mejores estrategias para informar de la zona geográfica, así como de elementos de elaboración, en especial de factores naturales y humanos; entre otros aspectos tradicionales (Europa Press, 2007). Mulder (2001) refiere en cuanto esto que:

El consumidor europeo busca cada vez más información sobre el origen y el proceso de elaboración de los productos alimenticios. Existen personas que prefieren productos auténticos que tengan más sabor y que sean elaborados de acuerdo a un conocimiento resguardado por la tradición. Ya no se trata de comparar productos estándares que se diferencian en precio, sino productos cuya calidad sea identificada, garantizada y que responda a lo esperado por el consumidor. Esta evolución del comportamiento de los consumidores europeos, junto con la saturación del mercado de los productos agroalimentarios ha contribuido fuertemente al desarrollo de una política favorable hacia la identificación cultural de los productos (Mulder, 2001, p. 10).

En general, lo antepuesto permite identificar el fortalecimiento de productos, que con el desarrollo y consolidación de estas prácticas, conven- cen al consumidor a mejorar su compra, comprometiendo indirectamente a productores a mejorar estándares de calidad relacionados con la procedencia del producto (Barquero, 2007).

Vale aclarar, que aunque el factor calidad no está siempre presente en las distinciones geográficas, cuando éstas se conciben dentro de las políticas de mejoramiento de la calidad agroalimentaria, constituyen sinónimo de calidad, que muy frecuente va más allá de la calidad básica referida a la sanidad e inocuidad, para convertirse en una "calidad superior" que se ofrece voluntariamente (MAPA, 1998).

Por ende supone garantizar la calidad y una forma eficaz contra las prácticas fraudulentas, siendo una de las fortalezas la aplicación de la "trazabilidad" o la producción integrada. Al respecto, Romero (2005) señala que a los productores rurales europeos:

(...) les ha correspondido poner todo ello en marcha para que el consumidor vea la autenticidad del producto en el mercado global, todo por no ver cómo su producto pierde cuota de mercado. Esto, o llegar a ser su presencia en el mercado tan solo de arraigo o testimonial siendo sustituido en práctica por otro producto distinto con el mismo nombre (p.15).

\section{Repoblamiento y rescate de suelos}

Las Indicaciones Geográficas no solo aumentan la producción sino, dan mayores oportunidades de trabajo local. En particular, los productores de Lentejas verdes de Puy ("Lentilles vertes du Puy"), solicitaron protección como Indicación Geográfica en Francia en 1990 y la cual desde que la obtuvieron en 1996, han tenido resultados significativos. No solo vieron aumentar la producción de 13600 quintales en 1990 a 34000 quintales en 1996 y 49776 quintales en 2002 (una progresión del 273\%) sino el número de ocupados, el cual se ha triplicado de 395 en 1990 a 750 en 1996 y 1079 en 2002 (Romero, 2005). 
La región española de Aragón, constituye una valiosa experiencia, pues parte de las iniciativas ligadas a las denominaciones geográficas han servido para retener población y crecer la renta de agricultores y ganaderos. No obstante, constituye ejemplo en que se evidencia el "renacimiento rural", con la consecuente renovación demográfica y el rescate de suelos. Así quedan en el pasado, espacios sometidos a la desertización, envejecimiento de población, al abandono de recursos territoriales y regresión económica (Romero, 2005).

Si bien, la región de Galicia es otro ejemplo del histórico abandono administrativo y problemas de despoblamiento, esa situación ha cambiado en últimos años, con el desarrollo de denominaciones geográficas u otras figuras de protección de calidad (Thual, s.f). Así se refiere un programa del canal internacional de televisión española: "Cerca de 1200 pueblos sometidos enteramente a la desolación, la deserción, en últimos años, solo esperan por la vida; así ven retroceder sus paisajes a una revitalización de la zona y el retorno a la vida" (Colom, 1995).

En general, el uso de estas herramientas se percibe en el ámbito socioeconómico, como excelente estrategia para mantener ocupada en labores propias del campo la abundante mano de obra. Quizás este puede ser uno de los logros más notables, al identificarse el rescate de la mano de obra, suelos, recursos endógenos como materias primas, que a diferencia de otrora, las poblaciones consideran aumentar la superficie cultivada a causa de la estabilidad de precios en el mercado, descenso de rentabilidad de otros cultivos y a creciente demanda y auge de productos con denominación dentro y fuera de la Unión Europea.

Romero (2005) verifica lo expuesto, cuando hace mención a publicación de la región española de Valencia del 23 de agosto 2005, en la que se refiere al aumento y rescate de superficie cultivada de Chufa Valenciana. Entre otros aspectos apunta a que...

(...) aumentará durante la campaña 20052006 entre un 15 y un 20 por ciento respecto al año pasado, por lo que su producción "batirá un récord histórico", según las previsiones del Consejo Regulador de la Denominación de Origen Chufa de Valencia. Los principales motivos del crecimiento de este producto son, según este Organismo, "la estabilidad de precio que encontró en el mercado unido en los últimos años, al descenso de rentabilidad de otros cultivos y a la creciente demanda de Chufa de Valencia con Denominación de Origen". Fuera de España se ha empezado a introducir "esta genuina bebida valenciana" en países como México, Panamá y la República Dominicana, explicaron las fuentes". (Romero, 2005).

Dentro de ese marco, un ejemplo lo constituye la producción del melón Castilla-La Mancha, España, que concentra el 35,70\% de superficie nacional de melón y el 31\% de la producción localizada principalmente en la provincia de Ciudad Real. El melón "piel de sapo" es uno de los cultivos característicos de comarca natural Mancha y supone complemento de renta de explotaciones familiares agrarias, cuyos principales ingresos proceden de la viña y cereales. Este carácter social viene también determinado por la pequeña dimensión de explotaciones, una media de 4,2 hectáreas. El cultivo genera en labores 235000 jornales al año y otros 265000 en recolección, manipulado y acondicionamiento para comercializar (Televisión Española Internacional, 2008).

\section{Protección cultural y recursos endógenos}

El patrimonio cultural o herencia colectiva incluye aspectos transmitidos de una generación a otra. Así, con las distinciones geográficas se valorizan elementos que han permitido construir culturas que incorporan cosmovisiones, lenguajes, formas de relación y sentidos de pertenencia e identidad, que determinan la forma de organización e inclusive la economía propia de un territorio rural (Romero, 2005).

Las comunidades enfatizan que el conocimiento tradicional es hereditario y debe protegerse du- 
rante el tiempo que perdure la cultura. En esa dirección, pueden mantenerse siempre y cuando la mezcla de las características culturales continúen.

En Francia, estas expresiones se han convertido en referentes que se deben proteger y mantener, para garantizar la autenticidad de productos con certificaciones, con denominaciones de origen y sellos de calidad, especialmente para muchos de sus quesos y su tradicional champaña. Con el afán de protegerlos se realizan actividades culturales, que sirven para promocionar productos de pequeñas comunidades. Para darle más realce e instituirlas participan todos los actores involucrados en el rubro de los productos (Gómez, 2003).

De este modo, mientras los métodos de producción pueden evolucionar a través del tiempo, el sistema de indicadores geográficos refleja un fuerte compromiso a las prácticas culturales derivadas de largos periodos de experiencia. Si bien, estas distinciones pueden servir como herramientas para que los poseedores del conocimiento tradicional se beneficien más equitativamente de la utilización comercial de su conocimiento; estos no monopolizan la utilización de cierta información, simplemente limitan las personas que pueden utilizar símbolos y prácticas culturales. Para Riveros y Blanco (2003):

(...) este tipo de distinciones se pueden considerar como expresiones que valoran la cultura del mundo rural, en el sentido, que también llevan asociado los recursos endógenos (paisaje, biodiversidad, emisión de oxígeno, permanencia de asentamientos humanos en zonas rurales, tradición, cultura) junto con la producción de bienes transables directamente (alimentos, bebidas, artesanías, confecciones, etc.) (Renamur, 2006).

\section{CONCLUSIONES}

Desde tiempos remotos, el medio rural ha significado un vital espacio, sin embargo, para la segunda mitad del siglo XX, se percibe el consiguiente desequilibrio territorial e infrautilización de los recursos naturales y ambientales. Ante la situación, no solo se reconoce la necesidad de mejorar la calidad de vida y de racionalizar el uso de los recursos naturales, sino indirectamente, favorecer el uso de denominaciones geográficas como instrumentos comerciales.

Por consiguiente, se perciben como valiosos instrumentos para el desarrollo y la promoción colectiva, ya que son fruto de relación estrecha entre grupos humanos y la tierra de donde provienen. En tanto, colaboran a promocionar y valorizar recursos, que entre otros beneficios reconocidos aproximan a comunidades desfavorecidas a la sostenibilidad y perdurabilidad ecológica, económica y social en general.

Así los mismos pasan a conformar puntos de referencia para encontrar nuevos papeles a la ruralidad ya que apuntan a ser sumamente beneficiosas, especialmente porque ayudan a proteger el valor local. En tanto inciden en el fortalecimiento de la posición de los productos autóctonos; al mantenerse cada región en lo que históricamente sabe mejor hacer; fomentando así, la cultura empresarial que persigue con sentido y eficacia aspiraciones comunes y relaciones de poder con otros agentes.

Entre otras razones destaca, que añaden valor a las tierras, en ese sentido, las estrategias por conservar y rescatar tierras hacen de las comunidades más dinámicas, a la vez, codiciadas y aptas para invertir. Así, localidades miradas con menospreciado, se tratan con potencial para desarrollar importantes proyectos de producción con protección, generando actividades económicas paralelas con sus consecuentes efectos de más empleo e ingresos.

En ese sentido, ayuda al repoblamiento y rescate de suelos al brindar mayores oportunidades de trabajo local y a retener población al crecer la renta. La consecuente renovación demográfica y el rescate de suelos, lo cual sirve de argumento adicional para arraigarse e integrar comunidades rurales, lo que conllevaba a crear un tejido social territorial articulado. Pues quedan en el 
pasado, espacios sometidos a la desertización, el envejecimiento de población, el abandono de recursos territoriales y la regresión económica.

En razón, las denominaciones geográficas ayudan a definir nuevas estrategias de mercado al apreciarse como nichos diferenciales de originalidad y seguridad. En particular, para competir e insertarse en la creciente globalización y mundialización económica y cultural. Así permite identificar el fortalecimiento de productos, que con el desarrollo y consolidación de estas prácticas, convencen al consumidor a mejorar su compra y comprometen indirectamente a productores a mejorar estándares de calidad relacionados con la procedencia del producto.

En general, el uso de estas herramientas se percibe en el ámbito socioeconómico, como excelente estrategia para mantener ocupada en labores propias del campo la abundante mano de obra. Protección cultural y recursos endógenos, en esa dirección. Las comunidades enfatizan que el conocimiento tradicional es hereditario y pueden mantenerse siempre y cuando la mezcla de las características culturales continúen.

\section{REFERENCIAS}

Ablan E. (2000). Políticas de Calidad en el Sistema Agroalimentario Español.Agroalimentaria (10), p. 63

Avelino, J., Marie, D. \& Ribeyre, F. (2007). Las indicaciones geográficas: una opción para agregarle valor al café. Centre de Coopération Internationale en Recherche Agronomique pour le Développement (CIRAD). Communication CIRAD. San Salvador, El Salvador.

Barquero, S. (2007, 20 de mayo). Starbucks mantiene a flote a 2.600 cafetaleros de Tarrazú. La Nación. Recuperado el 1-julio- 2008, de www.lanación. com San José, Costa Rica.

Bérard, L. \& Marchenay, P. (1996). La construcción social de los productos de la tierra. Agricultura y Sociedad, 80-81, pp. 31-56.

Caldentey, P. \& Gómez, A. C. (1996). Denominaciones de origen. Agricultura y Sociedad, 80-81, pp. 21-25

Caldentey, P. \& Gómez, A. C. (1997). Denominaciones de origen. Agricultura y Sociedad. 7 (31), pp. 30-32

Caldentey, P. \& Gómez, A. C. (2001, junio). Productos típicos y denominaciones: de la tutela a la divergencia.
IV Coloquio hispano-portugués de estudios rurales. Dpto. de Economía, Sociología y Política Agrarias. Universidad de Córdoba. Santiago de Compostela.

Caldentey, P. \& Gómez, A.C. (1998). Estudio para la implantación de signos de calidad en productos agroalimentarios de la Sierra Norte de Sevilla. Dpto. de Economía, Sociología y Política Agrarias. Universidad de Córdoba. Santiago de Compostela.

Cantarelli, F. (2000, Junio). El Observatorio Internacional para la valorización de los alimentos tradicionales de los países mediterráneos de la Unión Europea. Agroalimentaria, (10), pp. 9-10

Colom, A. \& Sáez, E. (1995). Desarrollo sostenible y perdurable en entornos desfavorecidos. Enfoque estratégico, mix de externalidades, pluriactividad y el papel de la formación y la divulgación. Curso Internacional de Desarrollo Rural y Vulgarización. CIHEAM-Montpellier- DARP. Reus.

Colom, A., Escardíbul B., Fransi C. \& Saez E. (1999). Algunas reflexiones ante la evolución rural en el nuevo milenio. Desarrollo rural y nuevas políticas estructurales. Universidad de Lleida, Facultad de Veterinaria, Escuela Técnica Superior de Ingeniería Agraria y Universidad de Zaragoza.

Comisión de la Unión Europea. (1998). Agenda 2000. Exposición de Motivos. Síntesis normativa. Unión Europea. Bruselas.

Comisión Europea. (2006). Política de Desarrollo Rural: 2007-2013. Agricultura y Desarrollo Rural. Recuperado el 10-marzo-2010 de ec.europea.eu/agriculture/rurdev/index

Davesne L. (2006, 5 de setiembre). De la importancia de las denominaciones de origen y de protección. Verema Interactiva. Recuperado el 10-marzo2010 de www.verema.com

Delgado, L. (1992). Análisis del comportamiento del consumidor: Técnicas multivariantes aplicadas al consumo de vino en Córdoba. Tesis de Doctorado. Universidad de Córdoba ETSIAM. Córdoba, España.

Delgado, M. M. \& Ramos, E. (2002). The role of European Rural Development Programmes in Enhancing Democracy: The Case of Southern Spain. En: A. Arzeni, R. Esspesti y F. Sotte. (Eds.). European Policy Experiences with Rural. Una publicación de Associazione Alessandro Bartola y European Association of Agricultural Economics. Kiel, KG.: Wissenschaftsvergag.

Delgado, M. M. \& Ramos, E. (2003). Understanding the evolution of the European Rural Policy: A methodological approach. Tesis de Doctorado. Departamento de Economía Agrícola, Escuela Superior 
de Ingenieros Agrónomos y Montes, Universidad de Córdoba. Córdoba, España.

Delgado, M. M. (2001). Análisis de los efectos de la nueva política rural europea: una aplicación al caso andaluz. Tesis de Doctorado. Departamento de Economía Agrícola, Escuela Superior de Ingenieros Agrónomos y Montes, Universidad de Córdoba. Córdoba, España.

Downes, D. R. \& Laird, S. A. (1997). Estudios de caso sobre Indicadores Geográficos y Marcas Comerciales. Gobierno de Noruega.

Europa Press. (2006, 6 de marzo). La Comisión Europea incluye al aceite de oliva de Monterrubio en sus denominaciones de origen protegidas. Bruselas. Recuperado el 12-setiembre-2008, de http://www. europapress.es/

Ferrari, J. P. (2008, 2 de agosto). La cariñena una cepa buscando identidad: originaria de España es hoy día un vino que empieza a resurgir. Estilos de Vida. 2 (655), pp. 11-13.

Fundación Eroski. ¿Qué son las denominaciones de origen?. España. Recuperado el 1-agosto-2008 de http://www.consumer.es

Gómez, A.C. \& Caldentey, P. (1999). Signos Distintivos en. Productos Agroalimentarios. Distribución y Consumo. 9 (45), pp. 71-84.

Gómez, A.C. \& Caldentey, P. (2000). Signos De Calidad En Productos Agroalimentarios. Tierra Sur. 8 (7), pp. 5-8.

Gómez, M. (2003, 20 de set). Denominaciones de origen e indicaciones geográficas. Secretaria de Agricultura de la Junta de las Comunidades de Castilla la Mancha. Castilla la Mancha, España. Recuperado el dd-mm-año de www.jccm.es.

Imprimireroski. (2002, 27 de agosto). Bruselas lanza una web sobre denominaciones de origen e indicaciones geográficas. Recuperado el 20 - junio2008, de www.Imprimireroski.com

La ruta dictada por el paladar. (2002, 8 de diciembre). El Financiero. San José, Costa Rica. Recuperado el 20-agosto 2008 de www.elfinancierocr.com

Llorente, M. (2001). La fuerza de la diferencia: la denominación de origen, un instrumento para el desarrollo. Huesca, España: Ediciones a Val de Onsera.

Marchenay, B. L. (1996). La construcción social de los productos de la tierra. Agriculturay Sociedad, 192 (1), p. 94.

Martínez M. P. \& Jiménez A. I. (1999). La potenciación del origen en las estrategias de marketing de productos agroalimentarios. Universidad de Castilla La Mancha y la Universidad Abierta de Cataluña. España.
Ministerio de Agricultura Pesca y Alimentación (MAPA). (1995, 1996, 1998, 2007). Madrid, España: Ministerio de Agricultura Pesca y Alimentación.

Moscoso, A. (1998). Importancia de la producción agroalimentaria de calidad en Europa y su influencia en el desarrollo rural: el papel de las denominaciones. Jornadas sobre Denominaciones de Origen. Departamento de Agricultura y Medio Ambiente, Gobierno de Aragón, Zaragoza, España.

Mulder J. (1998). Comisión Europea. Política de Calidad: Informe sobre una política de calidad para los productos agrarios y agroalimentarios. Recuperado el 21-julio-2008, de http:www.europa. eu.int/comm/agriculture/qual

Oficina de Enoturismo de Valladolid. Catas de vino. Recuperado el 9-marzo 2010, de www.valladolidenoturismo.com ó http://www.turismorural. com/Ficha.aspx?!D=595

Pérez, G. (1998). La denominación geográfica: su aplicación en la industria agroalimentaria tradicional española. Boletín Económico del ICE. (2563).

Renamur, G. (2007, 27 de abril). Algunos textos del curso sobre Denominaciones de Origen y bio territorio en México D.F. Mercado y productos Revista electrónica latinoamericana en Desarrollo Sustentable. Recuperado el 21-20 de febrero de 2010, de www.vinnculando.org.

Riveros, H. \& Blanco, M. (2003, Junio). Documento Técnico: El Agroturismo, una Alternativa para Revalorizar la Agroindustria Rural como Mecanismo de Desarrollo Local. Lima, Perú: IICA.PRODAR

Romero, A. (2005, 16 de setiembre). Horchata ¿De chufa...?. Agroterra. Recuperado el 8-marzo- 2010, de www.agroterra.com

Saraceno, E. (2000). La experiencia europea de desarrollo rural y su utilidad para el contexto latinoamericano. Taller sobre experiencias, políticas e instrumentos de desarrollo rural en los países latinos de Europa y América: un enfoque comparativo. San Fernando de Henares, Madrid, España.

Thual, D.. Denominaciones de Origen y marcas locales como medios paraincrementar el valorlocal. Secretaria General de Organisation for an International Geographical Indications Network ORIGIN.

TVE (2008,16 de agosto). Pueblos que esperan por la vida. Desde Galicia para el mundo. Programa de televisión transmitido por Televisión Española Internacional. 